\title{
Primary immunodeficiencies: not just paediatric diseases
}

\author{
Andrew R. Gennery ${ }^{1}$ and Steven M. Holland ${ }^{2}$ \\ Affiliations: ${ }^{1}$ Institute of Cellular Medicine, Newcastle University, Newcastle upon Tyne, UK. ${ }^{2}$ Laboratory of \\ Clinical Infectious Diseases, National Institute of Allergy and Infectious Diseases, NIH, Bethesda, MD, USA.
}

Correspondence: Andrew R. Gennery, Paediatric Immunology Dept, c/o Old Children's Outpatients, Great North Children's Hospital, Queen Victoria Road, Newcastle upon Tyne, NE1 4LP, UK.

E-mail: andrew.genneryancl.ac.uk

0

@ERSpublications

Childhood survivors of chronic granulomatous disease show the importance of expertise in primary immunodeficiencies http://ow.ly/K09mr

Primary immune deficiencies, once the sole domain of paediatricians, have come of age, and increasing numbers of childhood survivors, testament to meticulous medical care and the introduction of broad-spectrum antimicrobial prophylaxis, are now reaching early-to-mid adulthood. These patient cohorts are important for a number of reasons: 1) they teach us about the natural history of disease, modified through therapeutic intervention; 2) they highlight patients presenting at the extremes of the spectrum, e.g. with less severe disease, modified by genetics and/or environment; 3 ) they may offer insights into risks and benefits of earlier intervention in the disease course; and 4) they present a challenge to adult physicians, potentially unfamiliar with hitherto "paediatric" disease and its management.

Chronic granulomatous disease (CGD), a genetically inherited disorder of phagocyte function, is caused by mutations in genes encoding the five protein subunits of the NADPH oxidase, the enzyme responsible for the phagocyte respiratory burst and superoxide production [1,2]. The most common defects are in $\mathrm{X}$-linked gp91 ${ }^{\text {phox }}$, whilst defects in the other four protein components are inherited in an autosomal recessive fashion. The natural history of CGD is well understood. Patients with X-linked disease or those with absent or severely reduced superoxide production present earlier, have more severe disease and are most likely to die from complications of the disease [3, 4]. Major manifestations are of severe, sometimes fatal infections, predominantly from catalase-positive microorganisms, with Staphylococcus aureus, Burkholderia species and Aspergillus species being major pathogens [4-6]. Survival of CGD patients has improved with the introduction of antibacterial and antifungal prophylaxis $[4,6,7]$ and the use of interferon- $\gamma$ [8]. Inflammatory complications, particularly affecting the lungs and gastrointestinal tract, are also significant manifestations in patients; these are successfully managed with immunosuppression [4-7]. Increasingly, patients are being offered haematopoietic stem cell transplantation to cure the disease [9-11]. With such modern approaches, survival, for paediatric patients at least, is as good as survival for those taking antimicrobial prophylaxis [12]. The major issues, confronting patients and physicians alike, are which treatment option to follow and, if stem cell transplantation is to be offered, when is the most appropriate time to consider this. Although survival following stem cell transplantation, even for those patients who are sick at the time of transplantation [13], is good, a well-matched donor is not always available. Most transplants for CGD are performed in childhood or adolescence, but for those who choose not to be transplanted, or for whom a suitably matched donor is not available, how should physicians respond to the question "What is the outlook for adults with CGD today?"

The answer to this question is incomplete, but a study by SALVATOR et al. [14], in this issue of the European Respiratory Journal, goes some way to answering it. Using the national French registry for primary immunodeficiency, 99 adult patients out of 174 total patients with CGD were identified; complete

Received: Feb 042015 | Accepted: Feb 092015

Conflict of interest: None declared.

Copyright @ERS 2015 
data were available for 67 . The median duration of follow-up in adulthood (defined as age $>16$ years) was only 9 years, although the follow-up period ranged from 1 to 43 years, and the age of adult patients ranged from 17 to 59 years. Nine (13\%) out of the 67 patients died, predominantly from infection, most commonly due to Aspergillus species, the most common cause of death in CGD [15]. Respiratory complications (infection, particularly due to fungal species, or inflammation) were common, and more frequent than seen in childhood, despite prophylaxis. Treatment was complicated, often prolonged (>6 months) and involved lengthy hospitalisation and invasive, sometimes multiple therapies. Respiratory inflammatory complications occurred in more than a quarter of patients, although nonrespiratory complications were more likely, frequently involving the gastrointestinal tract. Noninfectious complications were more likely to be persistent.

This is an important study, because it begins to address some of the aforementioned issues. Whilst it is limited by the relatively small size of the cohort (although large in size for adult CGD populations), and is historic and retrospective in nature, nevertheless, important lessons can be drawn. First, many of these patients will have been treated in the "modern era", when prophylaxis with appropriate antifungal therapy and interferon- $\gamma$ will have been available; therefore, the results are relevant to patients who are making treatment decisions for themselves today. Secondly, many of the patients who were receiving appropriate antifungal prophylaxis still presented with fungal infections and were found to have subtherapeutic drug levels. This observation emphasises the difficulty of prophylaxis in this age group, either because of noncompliance or because gastrointestinal inflammation may impair absorption. The presumption that therapeutic drug levels would be more helpful in preventing infection is reasonable but unproven so far. Thirdly, this study highlights the importance of these patients being cared for in centres with broad experience in CGD, where adult physicians are aware of the peculiar characteristics of this patient group (for instance, the lack of symptoms in the presence of fungal pulmonary infection noted in this study) and are able to collaborate with physicians who have the most experience with the protean and confusing manifestations of this disease. As paediatric patients with rare diseases survive into adulthood, it will be increasingly important that appropriately skilled physicians are available to care for them, as has been the case for patients with cystic fibrosis [16].

Finally, within this cohort were a small number of patients who had previously undergone haematopoietic stem cell transplantation and who now presented with no symptoms, emphasising previous reports of the curative benefit of stem cell transplantation in abolishing infectious and inflammatory complications in this patient group. Stem cell transplantation can also normalise life quality for these patients [17]. Given the life-long risk and recurrence of respiratory infections and inflammatory sequelae that these patients experience, both in the respiratory and gastrointestinal tracts, the data presented in this article suggest that early consideration should be given to haematopoietic stem cell transplantation regardless of the age at which patients present, given the low toxicity and excellent survival of recent reports.

\section{References}

1 Roos D, Kuhns DB, Maddalena A, et al. Hematologically important mutations: X-linked chronic granulomatous disease (third update). Blood Cells Mol Dis 2010; 45: 246-265.

2 Roos D, Kuhns DB, Maddalena A, et al. Hematologically important mutations: the autosomal recessive forms of chronic granulomatous disease (second update). Blood Cells Mol Dis 2010; 44: 291-299.

3 Kuhns DB, Alvord WG, Heller T, et al. Residual NADPH oxidase and survival in chronic granulomatous disease. N Engl J Med 2010; 363: 2600-2610.

4 Jones LB, McGrogan P, Flood TJ, et al. Special article: chronic granulomatous disease in the United Kingdom and Ireland: a comprehensive national patient-based registry. Clin Exp Immunol 2008; 152: 211-218.

5 van den Berg JM, van Koppen E, Ahlin A, et al. Chronic granulomatous disease: the European experience. PLoS One 2009; 4: e5234.

6 Martire B, Rondelli R, Soresina A, et al. Clinical features, long-term follow-up and outcome of a large cohort of patients with chronic granulomatous disease: an Italian multicenter study. Clin Immunol 2008; 126: 155-164.

7 Mouy R, Fischer A, Vilmer E, et al. Incidence, severity, and prevention of infections in chronic granulomatous disease. J Pediatr 1989; 114: 555-560.

8 Marciano BE, Wesley R, De Carlo ES, et al. Long-term interferon-gamma therapy for patients with chronic granulomatous disease. Clin Infect Dis 2004; 39: 692-699.

9 Horwitz ME, Barrett AJ, Brown MR, et al. Treatment of chronic granulomatous disease with nonmyeloablative conditioning and a T-cell-depleted hematopoietic allograft. N Engl J Med 2001; 344: 881-888.

10 Seger RA, Gungor T, Belohradsky BH, et al. Treatment of chronic granulomatous disease with myeloablative conditioning and an unmodified hemopoietic allograft: a survey of the European experience, 1985-2000. Blood 2002; 100: 4344-4350.

11 Soncini E, Slatter MA, Jones LB, et al. Unrelated donor and HLA-identical sibling haematopoietic stem cell transplantation cure chronic granulomatous disease with good long-term outcome and growth. $\mathrm{Br} \mathrm{J} \mathrm{Haematol}$ 2009; 145: 73-83.

12 Cole T, Pearce MS, Cant AJ, et al. Clinical outcome in children with chronic granulomatous disease managed conservatively or with hematopoietic stem cell transplantation. J Allergy Clin Immunol 2013; 132: 1150-1155. 
13 Güngör T, Teira P, Slatter M, et al. Reduced-intensity conditioning and HLA-matched haemopoietic stem-cell transplantation in patients with chronic granulomatous disease: a prospective multicentre study. Lancet 2014; 383: 436-448.

14 Salvator $\mathrm{H}$, Mahlaoui $\mathrm{N}$, Catherinot $\mathrm{E}$, et al. Pulmonary manifestations in adult patients with chronic granulomatous disease. Eur Respir J 2015; 45: 1613-1623.

15 Marciano BE, Spalding C, Fitzgerald A, et al. Common severe infections in chronic granulomatous disease. Clin Infect Dis 2015; 60: 1176-1183.

16 Stephenson AL, Tom M, Berthiaume Y, et al. A contemporary survival analysis of individuals with cystic fibrosis: a cohort study. Eur Respir J 2015; 45: 670-679.

17 Cole T, McKendrick F, Titman P, et al. Health related quality of life and emotional health in children with chronic granulomatous disease: a comparison of those managed conservatively with those that have undergone haematopoietic stem cell transplant. J Clin Immunol 2013; 33: 8-13. 